\title{
Rare variant collapsing in conjunction with mean $\log p$-value and gradient boosting approaches applied to Genetic Analysis Workshop 17 data
}

\author{
Yauheniya Cherkas ${ }^{1}$, Nandini Raghavan², Stephan Francke ${ }^{3}$, Frank DeFalco ${ }^{4}$, Marsha A Wilcox ${ }^{1 *}$ \\ From Genetic Analysis Workshop 17 \\ Boston, MA, USA. 13-16 October 2010
}

\begin{abstract}
In addition to methods that can identify common variants associated with susceptibility to common diseases, there has been increasing interest in approaches that can identify rare genetic variants. We use the simulated data provided to the participants of Genetic Analysis Workshop 17 (GAW17) to identify both rare and common singlenucleotide polymorphisms and pathways associated with disease status. We apply a rare variant collapsing approach and the usual association tests for common variants to identify candidates for further analysis using pathway-based and tree-based ensemble approaches. We use the mean log p-value approach to identify a top set of pathways and compare it to those used in simulation of GAW17 dataset. We conclude that the mean log pvalue approach is able to identify those pathways in the top list and also related pathways. We also use the stochastic gradient boosting approach for the selected subset of single-nucleotide polymorphisms. When compared the result of this tree-based method with the list of single-nucleotide polymorphisms used in dataset simulation, in addition to correct SNPs we observe number of false positives.
\end{abstract}

\section{Background}

Many genome-wide association studies (GWAS) have been conducted in the search for specific genetic variants associated with common diseases. In testing for association with common polymorphisms, those variants that were identified were able to explain a modest proportion of disease heritability. This led to the hypothesis that multiple rare variants may play a role in complex disease etiology [1][2][3]. The multiple rare variants or common disease/rare variant hypothesis states that multiple rare variants with moderate to high penetrances underlie the susceptibility to a common disease. It is likely that both common and rare genetic variants contribute to disease risk.

Approaches targeted at uncovering associations between common polymorphisms and disease are generally underpowered for detecting the influence of rare

\footnotetext{
* Correspondence: mwilcox@its.jnj.com

'Epidemiology, Johnson \& Johnson, 1125 Trenton-Harbourton Road,

Titusville, NJ 08560, USA

Full list of author information is available at the end of the article
}

variants. To identify disease-associated rare variants, investigators have proposed several methods based on the collapsing of low-frequency single-nucleotide polymorphisms (SNPs) [4-7].

In this analysis we use the methods proposed by $\mathrm{Li}$ and Leal [4] and Morris and Zeggini [8] to identify rare variants, and we use association analysis to identify common variants that confer liability to disease. The rationale behind this collapsing approach is that although the probability that an individual carries more than one rare allele may be low, in aggregate rare alleles may be common enough to account for variation in common traits. The goal is then to test for an association of an accumulation of rare minor alleles with the disease trait, by combining information across multiple variant sites.

We begin our analyses with the collapsing methods and extend the analyses in two ways. First, we use the mean $\log p$-value (MLP) [9], which is a method that takes into account information about SNP function and ontologic pathway. The MLP can be thought of as a 
way to group together SNPs by their functional implication. It was originally developed for the analysis of gene expression data for a better understanding of the underlying mechanisms. Thus, by further analyzing the results of the rare variant collapsing approach using MLP analysis, we exploit both the spatial and the functional associations of SNPs implicated in a disease. Second, we use an empirical approach, stochastic gradient boosting (SGB), to discern groups of SNPs conferring liability to disease. SGB is an ensemble tree-based method [10] that is useful for empirically detecting sets of genes associated with a disease.

\section{Methods}

Data

Our analyses focus on the case-control data provided to the participants of Genetic Analysis Workshop 17 [11]. We selected the first of 200 simulated replicates for analysis. We conducted the Hardy-Weinberg equilibrium test using PLINK [12] and excluded from further analysis all markers with deviations ( $p$-value less than 0.0001 in control subjects). We conducted population stratification analysis by first excluding correlated markers and then using the multidimensional scaling methods in PLINK.

\section{Significant markers}

We use the collapsing method proposed by Li and Leal [4] and Morris and Zeggini [8] to identify possible variants among rare SNPs. For this analysis we use the CCRaVAT (Case-Control Rare Variant Analysis Tool) software package [13]. The collapsing method is as follows: first, we divide the markers into groups on the basis of predefined criteria (either genes or sliding windows of defined sequence length); next, we collapse marker data based on an indicator variable that shows whether a subject carries any rare variants; and finally, using a Pearson chi-square test, we test the significance of the difference in proportions between case subjects and control subjects who carry rare variant minor alleles. When cell counts are small, we use a Fisher exact test instead.

We consider several approaches for the collapsing criterion, including gene-based collapsing and sliding windows of five different sizes $(1 \mathrm{~kb}, 5 \mathrm{~kb}, 25 \mathrm{~kb}, 50 \mathrm{~kb}$, and $100 \mathrm{~kb})$. The resulting $p$-values are recorded for further analysis. In addition, we test the common variant SNPs using the Pearson chi-square test. Again, the resulting $p$-values are retained for further analysis.

\section{MLP approach}

We use the MLP approach [9] to incorporate functional and pathway information about genes into our analysis. The MLP analysis was developed in the context of gene expression analysis. The idea is to first assign a statistic (e.g., a $p$-value) to each gene. The genes are mapped onto gene sets or pathways by utilizing gene annotation databases, such as the Kyoto Encyclopedia of Genes and Genomes (KEGG) [14], the Ingenuity Pathway Analysis (IPA) [15], and the Gene Ontology (GO) Biological Processes databases [16]. Permutation tests are used to determine a $p$-value for a gene set and to identify the top set of gene sets.

In our analysis, we explore both rare and common variants. To assign a $p$-value to a gene, we use the results of collapsing and association tests. Thus a gene can have multiple $p$-values associated with it, especially in the rare variant analysis, because the windows overlap. We examine several ways to assign the $p$-value to a gene in order to explore the utility of each, three for rare variants and two for common variants. For rare variants, we use (1) the $p$-value from the association test based on gene-wise collapsing or (2) the minimum $p$ value among association tests based on the collapsing within 5 -kb sliding windows located in a gene. This window size is based on the preliminary explorations of varying window sizes ranging from $1 \mathrm{~kb}$ to $100 \mathrm{~kb}$. In addition, we use (3) the mean $\log p$-value among association tests based on the collapsing within $5-\mathrm{kb}$ sliding windows located in a gene. For common variants, we use either (1) the minimum $p$-value among SNPs in a gene or (2) the mean $\log p$-value among SNPs in a gene.

We create gene sets, consisting of groups of genes, on the basis of one of the databases (KEGG, IPA, or GO). The gene set statistic is subsequently calculated as the MLP of the gene statistics for each gene set. The permutation procedure described by Raghavan et al. [9] is used to obtain the gene set $p$-value. The gene sets are rank-ordered by $p$-value. We examine the top 20 sets and present the top 6 sets in this report.

\section{Stochastic gradient boosting}

SGB [10] is an ensemble tree-based method that uses an independently drawn random sample of individuals and SNPs to construct a small tree, typically containing 2 to 12 terminal nodes. The tree is grown as a result of recursively partitioning a node and contributes a small portion to the overall model. Each consecutive tree is built for the prediction residuals (from all preceding trees) of an independently drawn random sample. The final SGB model and its prediction perform by combining weighted individual tree contributions, with weight being a shrinkage parameter appropriately selected to reduce overfitting.

The SGB method produces a variable importance measure that can be used to identify top predictors. For tree methods variable importance scores show the relative contribution of each of the variables to predicting 
the outcome. For ensemble methods, such as SGB, the variable importance scores are averaged across all trees.

We apply the SGB method, using TreeNet, developed by Salford Systems [17], to the data consisting of the first replicate of the affected phenotype, multidimensional scaling components, environmental predictors (Age, Smoke, and Sex), and SNPs. We start with a set of the top common and rare SNPs passed from the collapsing approach and association tests. We then use the set of all SNPs provided in the GAW17 dataset.

\section{Results}

The initial data analysis of minor allele frequencies (MAFs) in the case-control data showed 3,224 rare variants (MAF between $1 \%$ and $5 \%$ ) and 18,131 very rare variants (MAF less than 1\%). There were 209 (30\%) affected case subjects and 488 (70\%) unaffected control subjects. Twenty-nine percent of males and thirty-one percent of females were affected. Smoking differed with case status. Smoking was prevalent in $35.9 \%$ of the affected subjects, in contrast to $21.7 \%$ of the unaffected subjects. After filtering, the data set included 22,615 SNPs. The population stratification results (the first two components) are shown in Figure 1. Three clusters were identified, corresponding to three populations. The resulting dimensions were carried forward for stratification.

We performed collapsing for various window sizes (1 $\mathrm{kb}, 5 \mathrm{~kb}, 25 \mathrm{~kb}, 50 \mathrm{~kb}$, and $100 \mathrm{~kb}$ ) as well as gene-wise collapsing. The Manhattan plot of $p$-values produced by the collapsing approach for $5-\mathrm{kb}$ sliding windows is

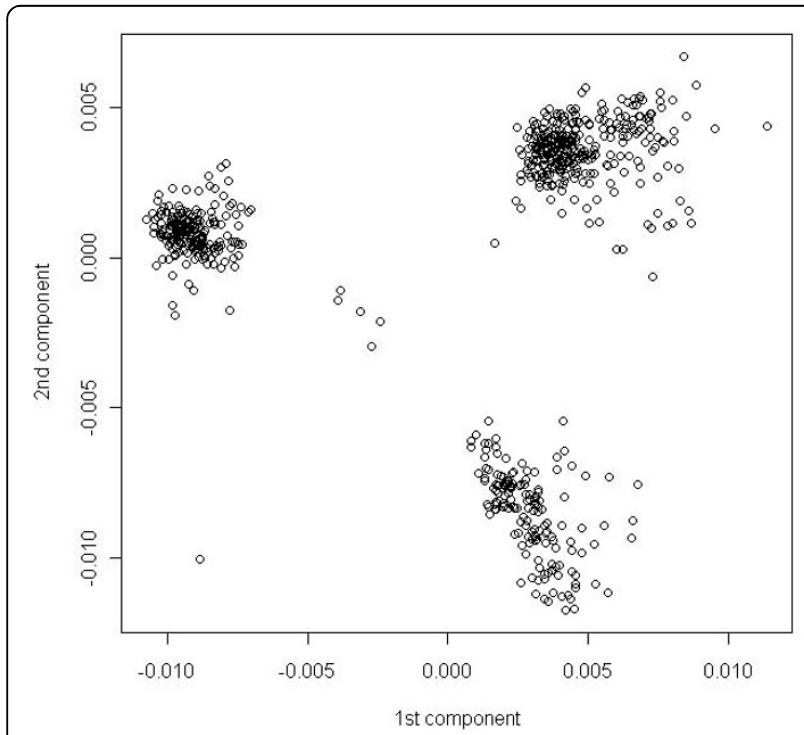

Figure 1 Plot of the first two components of multidimensional scaling shown in Figure 2. The results from the 5-kb analysis were carried into further analyses.

We used the MLP approach to identify the top gene sets based on the KEGG, IPA, and GO databases. We examined the results from the MLP analysis using IPA pathways in greater detail, because this was the database used to simulate the GAW17 data. The top 20 gene sets were examined. Results for the top six sets are summarized in Table 1. The three gene statistics for rare variants and the two gene statistics for common variants described in the "MLP Approach" subsection of the Methods section are presented. The top gene sets based on the minimum of window $p$-values shows the Notch, Hypoxia, Nitric Oxide, and vascular endothelial growth factor (VEGF) signaling pathways. Both the VEGF and the Notch signaling pathways control initiation and differentiation in angiogenesis, a process leading to blood vessel formation or remodeling. The VEGF pathway is also among the top 15 pathways identified using the KEGG database and the same statistic (results not shown).

We applied the SGB approach to the data containing preselected SNPs, population stratification results, and environmental variables. We used TreeNet to perform the SGB. We built 5,000 trees (iterations) with a maximum of 8 nodes. We chose a shrinkage parameter of 0.01 as appropriate for a data set of this dimension $[18,19]$. The top set of SNPs was selected using a variable importance measure of 7.00 as a cutoff threshold. The corresponding genes were also recorded. The results of the SGB approach are shown in Table 2. The table contains the top SNPs selected and their corresponding genes. The results that match the simulated model are shown in bold. The SGB analyses using the complete set of SNPs did not show an improvement over prior runs (results not shown).

The MLP approach correctly placed the VEGF signaling pathway and the two pathways related to the cardiovascular system (Hypoxia and Nitric Oxide) among the top six pathways. There are only 5 (out of 216) SNPs correctly identified using the SGB approach; their MAFs range from $0.2 \%$ to $17 \%$. Most of the SNPs (211) placed in the top list are false positives.

\section{Discussion and conclusions}

Traditionally, GWAS test for association of disease with common polymorphisms. Polymorphisms with population frequencies of $5 \%$ or more could be tested directly or indirectly for association with disease risk or quantitative traits, and GWAS have identified many genetic variants associated with disease traits. Replication of these results has not been consistently successful. More recently, methods have been proposed to identify multiple rare variants with small individual effect sizes that 


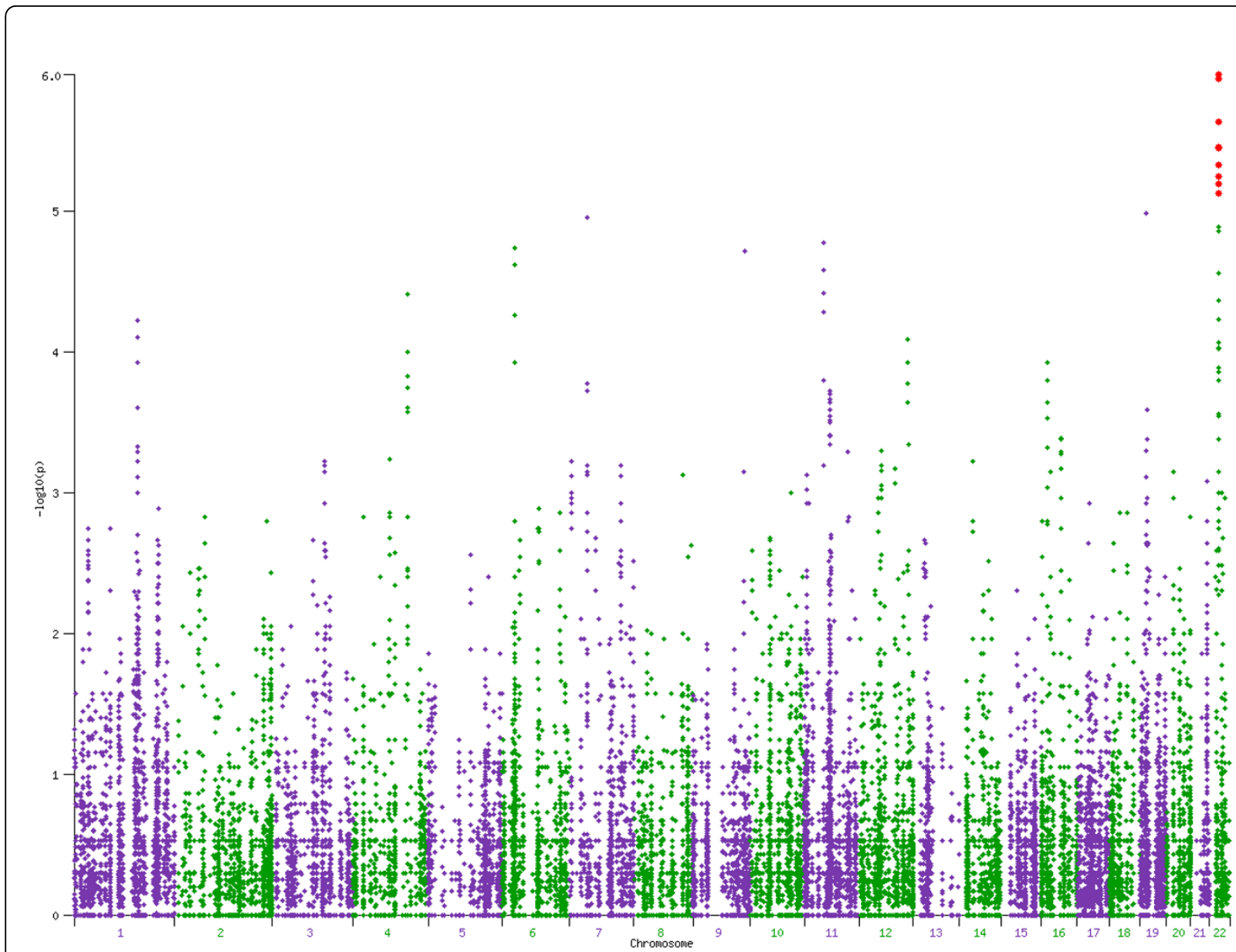

Figure 2 Manhattan plot of collapsing approach $p$-values for 5-kb sliding window

may be implicated in complex multigenic diseases. These methods are based on grouping rare variants by their physical proximity in order to combine information across them. According to Hirschhorn [20], one of the primary goals of GWAS is to discover the biologic pathways underlying polygenic diseases.

The MLP method is an effort in this direction, where both common and rare variants are considered on the basis of their functional implication in disease etiology. Our goal here was to exploit both the spatial and the functional associations of SNPs implicated in a disease to identify the underlying biologic pathways. Pathways used to simulate the affection status in the GAW17 data set were among the top four pathways identified by the MLP approach based on statistic 2 for rare variants. More specifically, the three pathways (Hypoxia, Nitric Oxide, and VEGF) were used to simulate the data. In addition, as explained in what follows, the top four pathways, including the Notch pathway, may be part of a cascade of interrelated pathways. Enriched signaling pathways in our analysis may overlap functionally and indicate processes leading to angiogenesis. Hypoxia signaling can trigger the VEGF cascade in cancer tissue angiogenesis, and the Notch processes downstream from the Hypoxia and VEGF pathways lead to a differentiation of newly formed vessels. Notch signaling can also down-regulate VEGF expression in a feedback loop. Thus the MLP approach based on statistic 2 for rare variants appears to be able to also identify related pathways and may be promising for the discovery of biological pathways implicated in disease etiology by rare variants.

One of the goals of this analysis was to compare results from a variety of functional and pathway databases and from a number of gene statistics. Our results indicate that using the IPA database, the gene statistic that identified the most relevant pathways was the minimum $p$-value derived from collapsing rare variants within $5-\mathrm{kb}$ sliding windows residing in a gene. These results highlight the importance of using the most appropriate pathway database for the analysis, an aspect not explicitly discussed in the literature. Our analysis 
Table 1 Major IPA pathways identified by the MLP approach using five statistics for rare and common variants and their corresponding $p$-values

\begin{tabular}{|c|c|c|}
\hline Statistic & Top 6 pathways selected & $p$-value \\
\hline \multicolumn{3}{|l|}{ Rare variants } \\
\hline \multirow[t]{6}{*}{ Gene-wise collapsing } & 1. Androgen and estrogen metabolism & 0.0006 \\
\hline & 2. Sphingolipid metabolism & 0.0006 \\
\hline & 3. Phenylalanine metabolism & 0.0015 \\
\hline & 4. Death receptor signaling & 0.002 \\
\hline & 5. Stilbene, coumarine, and lignin biosynthesis & 0.0036 \\
\hline & 6. TWEAK signaling & 0.0043 \\
\hline \multirow[t]{6}{*}{ Minimum $p$-value of 5-kb sliding window collapsing within a gene } & 1. Notch signaling & 0.0248 \\
\hline & 2. Hypoxia signaling in the cardiovascular system & 0.0403 \\
\hline & 3. Nitric oxide signaling in the cardiovascular system & 0.0409 \\
\hline & 4. VEGF signaling & 0.0585 \\
\hline & 5. Glutamate receptor signaling & 0.0642 \\
\hline & 6. Glutamate metabolism & 0.0741 \\
\hline \multirow[t]{6}{*}{ Mean log $p$-value of 5-kb sliding window collapsing within a gene } & 1. Cyanoamino acid metabolism & 0.0032 \\
\hline & 2. Ubiquinone biosynthesis & 0.0148 \\
\hline & 3. Nitrogen metabolism & 0.0267 \\
\hline & 4. Alanine and aspartate metabolism & 0.0392 \\
\hline & 5. GABA receptor signaling & 0.0423 \\
\hline & 6. FXR/RXR activation & 0.0438 \\
\hline \multicolumn{3}{|l|}{ Common variants } \\
\hline \multirow[t]{6}{*}{ Minimum $p$-value among SNPs } & 1. Apoptosis signaling & 0.0083 \\
\hline & 2. Pyrimidine metabolism & 0.0232 \\
\hline & 3. CNTF signaling & 0.0429 \\
\hline & 4. FLT3 signaling in hematopoietic progenitor cells & 0.0601 \\
\hline & 5. Role of NANOG in mammalian embryonic stem cell pluripotency & 0.0847 \\
\hline & 6. EGF signaling & 0.0908 \\
\hline \multirow[t]{6}{*}{ Mean log $p$-value for SNPs in a gene } & 1. Pyrimidine metabolism & 0.0021 \\
\hline & 2. CNTF signaling & 0.0127 \\
\hline & 3. Melanocyte development and pigmentation signaling & 0.0221 \\
\hline & 4. JAK/Stat signaling & 0.0327 \\
\hline & 5. IL-15 signaling & 0.0356 \\
\hline & 6. FLT3 signaling in hematopoietic progenitor cells & 0.045 \\
\hline
\end{tabular}

also indicates that (1) the results can be influenced by the density of coverage of rare variant SNPs in a gene; (2) gene-based collapsing may be too broad and may dilute the underlying information; and (3) using the mean of the window $p$-values may mute the signal considerably. In future work, we will evaluate alternative approaches to mapping SNP-level $p$-values to gene-level $p$-values as well as methods for combining the rare and common variants analyses.

The top pathways identified using the MLP method intersect with the pathways that contain genes from the results of the SGB approach. There are 5 correct SNPs out of 216 residing in 5 correct genes out of 188 corresponding to the top selected SNPs. The large number of false positives may be due to correlation of the SNPs identified by the SGB approach with the true SNPs used in the simulation model. Our current work includes studying methods to bridge the two approaches utilizing the functional information and the statistical correlation, respectively.

Currently, many analytic strategies rely on GWAS with one-SNP-at-a-time analyses. Although this approach has certainly yielded many promising candidates, it requires large samples to mitigate type I errors. One-SNP-at-a-time analyses generally do not take advantage of all the information present in the data, and failure of replication is commonplace. Recent attempts have been made to incorporate information from rare variants into the analysis by aggregating across SNPs that are in close proximity to each other. We have extended this further by leveraging information from SNPs that are either functionally related (MLP 
Table 2 Top SNPs and corresponding genes identified using the SGB approach

\begin{tabular}{|c|c|c|c|c|c|c|c|c|}
\hline \multirow[b]{2}{*}{ SNPs } & \multicolumn{8}{|c|}{ Top SNPs identified (from highest to lowest variable importance) } \\
\hline & C13S523 & C9S3621 & C6S6142 & C5S237 & C9S4860 & C22S1374 & C2S5630 & C11S60 \\
\hline & C13S905 & C1S6542 & C7S2893 & C19S282 & C6S1097 & C10S2632 & C2S955 & C145784 \\
\hline & C1S5779 & C7S2446 & C9S1469 & C12S4668 & C2S1087 & $\mathrm{C} 2 \mathrm{~S} 2148$ & C1S10506 & C6S2129 \\
\hline & C15S3343 & C12S4188 & C14S1863 & C2S4601 & C6S2469 & C10S2533 & C19S609 & C10S5515 \\
\hline & C1S5530 & C17S3017 & C9S1225 & C13S522 & C12S3028 & C5S3461 & C19S1762 & C1S9584 \\
\hline & C19S1849 & C9S4013 & C22S1405 & C12S622 & C12S7056 & C2S7558 & C16S3421 & C12S552 \\
\hline & C2S4407 & C1S996 & C22S1351 & C20S2310 & C22S1158 & C15S4060 & C17S1262 & C3S1305 \\
\hline & C7S158 & C10S387 & C17S2377 & C7S1877 & C1S9718 & C10S4422 & C4S2872 & C7S3971 \\
\hline & C2S689 & C8S3322 & C10S6566 & C14S20 & C7S1076 & C11S3224 & C1S7413 & C22S146 \\
\hline & C8S4238 & C8S4028 & C18S2322 & C6S6040 & C12S5220 & C6S6177 & C19S3382 & C19S2528 \\
\hline & C1S9506 & C4S4283 & C12S3528 & C11S2585 & C17S2376 & C12S5446 & C17S4841 & C1S10200 \\
\hline & C4S2239 & C7S3613 & C5S4072 & C11S6503 & C11S4881 & C1S10800 & C9S123 & C2S7414 \\
\hline & C2S1139 & C3S3962 & C7S3490 & C10S5783 & C11S1683 & C9S2613 & C11S2532 & C7S4111 \\
\hline & C18S2310 & C2S4079 & C6S2366 & C8S627 & C2S6985 & C1S7941 & C11S5292 & C4S4339 \\
\hline & C3S3938 & C6S5380 & C22S875 & C1S7092 & C7S2590 & C11S2871 & C6S2216 & C6S5677 \\
\hline & C7S4646 & C8S850 & C8S271 & C4S2296 & C10S386 & C9S51111 & C15S3138 & C1S7427 \\
\hline & C17S3510 & C3S96 & C22S385 & C1S3900 & C3S4638 & C21S672 & C1S1388 & C10S2683 \\
\hline & C13S1168 & C7S3697 & C2S4909 & C11S1280 & C2S2154 & C12S4591 & C3S1176 & C22S2039 \\
\hline & C11S3320 & C2S873 & C9S3100 & C2S7390 & C12S5526 & C11S1599 & C6S4552 & C1S10256 \\
\hline & C10S3243 & C12S5510 & C4S2678 & C4S2970 & C2S8207 & C16S560 & C6S7138 & C17S321 \\
\hline & C20S1844 & C12S5445 & C10S2670 & C1S4009 & C17S2026 & C9S3554 & C13S1660 & C14S590 \\
\hline & C10S6432 & C9S759 & C19S4625 & C1S9511 & C8S934 & C6S4242 & C18S1560 & C4S97 \\
\hline & C15S3744 & C7S397 & C19S4658 & C12S4534 & C9S2083 & C19S5271 & C7S3898 & C1S4838 \\
\hline & C9S1607 & C4S3834 & C11S5644 & C15S2848 & C10S3777 & C3S3657 & C14S122 & C14S3426 \\
\hline & C16S1482 & C4S3076 & C9S2542 & C2S6995 & C21S778 & C9S1835 & C15S3559 & C8S3416 \\
\hline & C5S2032 & C1S10813 & C10S5690 & C1S3676 & C6S4400 & C13S163 & C22S645 & C12S3039 \\
\hline & C1S10164 & C6S7164 & C22S1222 & C4S649 & C19S277 & C1S7408 & C1S1542 & C4S186 \\
\hline \multirow[t]{24}{*}{ Genes } & TNFRSF25 & AHSA2 & $A D H 1 B$ & GPR85 & OR13C5 & SYTL2 & PSME2 & PTPRS \\
\hline & ARHGEF1OL & RGPD3 & C4ORF33 & PTPRZ1 & CDK5RAP2 & PDGFD & $V T I 1 B$ & $\mathrm{OR} 1 \mathrm{OH} 3$ \\
\hline & KIF17 & RGPD4 & TKTL2 & PAX4 & STOM & EXPH5 & BEGAIN & CYP4F2 \\
\hline & PDE4B & ACVR1C & ANP32C & SMO & GOLGA1 & OR8D4 & PAQR5 & ZNF486 \\
\hline & PTGER3 & LY75 & PLEKHG4B & $\mathrm{ZC} 3 \mathrm{HCl}$ & BRD3 & CLEC2D & TSPAN3 & NPHS1 \\
\hline & MSH4 & $P P I G$ & ZNF474 & $A K R 1 B 1$ & CARD9 & $K L R K 1$ & ADAMTS7 & ZNF576 \\
\hline & STXBP3 & WDR75 & ABLIM3 & SLC37A3 & ECHDC3 & OR6C1 & ALPK3 & LYPD5TMC4 \\
\hline & HIPK1 & LOC729332 & FOXI1 & GATA4 & ERCC6 & OR6C65 & SLC28A1 & C20ORF32 \\
\hline & VTCN1 & UGT1A10 & $P G B D 1$ & TNFRSF10D & PGBD3 & SRGAP1 & AKAP13 & PRIC285 \\
\hline & $A R N T$ & COL6A3 & BAT2 & CDCA2 & $H K D C 1$ & PLXNC1 & ZNF213 & PIGP \\
\hline & ADAM15 & MTERFD2 & SLC44A4 & PTK2B & MAT1A & C12ORF63 & USP31 & ETS2 \\
\hline & OR10J1 & CRELD1 & PSMB8 & EXT1 & CYP2C8 & CHPT1 & LOC100132786 & HIRA \\
\hline & OR10J5 & LOC100130135 & MDN1 & SAMD12 & SORCS1 & TRAFD1 & TRPV3 & ARVCF \\
\hline & UAP1 & SETD2 & VNN1 & MLZE & CASP7 & CAMKK2 & KCNJ12 & TOP3B \\
\hline & LRRN2 & GOLGBI & FUCA2 & TG & DCLREIA & ANAPC5 & SLFN13 & SUSD2 \\
\hline & NUAK2 & TMCC1 & UTRN & ANKRD15 & TACC2 & ZNF26 & PIP4K2B & SEC14L3 \\
\hline & IKBKE & TRPC1 & AGPAT4 & PDCD1LG2 & ATHL1 & TNFRSF19 & $B R C A 1$ & SMTN \\
\hline & LAMB3 & CRIPAK & PMS2 & AQP3 & GALNTL4 & FLT1 & FAM117A & LIMK2 \\
\hline & DUSP10 & GRK4 & TSPAN13 & C9ORF131 & HPS5 & TRPC4 & RECQL5 & LOC100132621 \\
\hline & KIAA0133 & AFAP1 & NPC1L1 & NPR2 & NELL1 1 & FREM2 & HRH4 & \\
\hline & ARLGIP2 & PF4V1 & NCF1 & POLRIE & $\mathrm{OR} 8 \mathrm{H} 1$ & PIBF1 & B4GALT6 & \\
\hline & OXER1 & STBD1 & $F B X O 24$ & SMC5 & OR9G4 & RNASE6 & MCART2 & \\
\hline & FSHR & FAM13A1 & FBXL13 & C9ORF79 & OR4D9 & FLJ10357 & ZNF57 & \\
\hline & $B C L 11 A$ & PDLIM5 & RELN & ROR2 & AHNAK & ACIN1 & ZNF77 & \\
\hline
\end{tabular}


approach) or statistically correlated (SGB approach) with the hope of obtaining results that are credible and logically interpretable. These methods would, of course, need to be further evaluated in other data sets and other settings.

\section{Acknowledgments}

The Genetic Analysis Workshop is supported by National Institutes of Health grant R01 GM031575. We are grateful to Jesse Berlin and Paul Stang for their help with access to the data and resources. We would also like to thank Qingqin Li and Dai Wang for several discussions about the design and methods we used.

This article has been published as part of BMC Proceedings Volume 5 Supplement 9, 2011: Genetic Analysis Workshop 17. The full contents of the supplement are available online at http://www.biomedcentral.com/1753$6561 / 5$ ? issue $=$ S9.

\section{Author details}

'Epidemiology, Johnson \& Johnson, 1125 Trenton-Harbourton Road, Titusville, NJ 08560, USA. ${ }^{2}$ Non-Clinical Biostatistics, Johnson \& Johnson, OMP Building, 1000 Route 202-S, Raritan, NJ 08869, USA. ${ }^{3}$ Pharmacogenomics, Johnson \& Johnson PRD, PO Box 300, 1000 Route 202, Raritan, NJ 08869, USA. ${ }^{4}$ Informatics, Johnson \& Johnson, 920 Route 202, Raritan, NJ 08869, USA

\section{Authors' contributions}

YC carried out the collapsing approach, pathway and tree-based analyses and participated in writing the manuscript. MW, NR, SF designed the analysis plan and participated in writhing the manuscript. FD prepared the data for analysis and edited the manuscript. All authors read and approved the final manuscript.

\section{Competing interests}

The authors declare that there are no competing interests.

Published: 29 November 2011

\section{References}

1. Bodmer W, Bonilla C: Common and rare variants in multifactorial susceptibility to common diseases. Nat Genet 2008, 40:695-701.

2. Pritchard JK: Are rare variants responsible for susceptibility to complex diseases? Am J Hum Genet 2001, 69:124-137.

3. Pritchard JK, Cox NJ: The allelic architecture of human disease genes: common disease-common variant ... or not? Hum Mol Genet 2002, 11:2417-2423.

4. Li B, Leal S S: Novel methods for detecting associations with rare variants for common diseases: application to analysis of sequence data. Am J Hum Genet 2008, 83:311-321.

5. Li Q, Zhang H, Yu K: Approaches for evaluating rare polymorphisms in genetic association studies. Hum Hered 2010, 69:219-228.

6. Madsen BE, Browning SR: A groupwise association test for rare mutations using a weighted sum statistic. PLOS Genet 2009, 5:e1000384.

7. Morgenthaler S, Thilly WG: A strategy to discover genes that carry multiallelic or mono-allelic risk for common diseases: a cohort allelic sums test (CAST). Mutat Res 2007, 615:28-56.

8. Morris AP, Zeggini E: An evaluation of statistical approaches to rare variant analysis in genetic association studies. Genet Epidemiol 2010 34:188-193.

9. Raghavan N, Amaratunga D, Cabrera J, Nie A, Qin J, McMillian M: On methods for gene function scoring as a means of facilitating the interpretation of microarray results. J Comput Biol 2006, 13:798-809.

10. Friedman JH: Stochastic gradient boosting. Comput Stat Data Anal 2002, 38:367-378.

11. Almasy LA, Dyer TD, Peralta JM, Kent JW Jr., Charlesworth JC, Curran JE, Blangero J: Genetic Analysis Workshop 17 mini-exome simulation. BMC Proc 2011, 5(suppl 9):S1.

12. Purcell $S$, Neale B, Todd-Brown K, Thomas L, Ferreira MA, Bender D, Maller J, Sklar P, de Bakker PI, Daly MJ, Sham PC: PLINK: a tool set for whole- genome association and population-based linkage analyses. Am J Hum Genet 2007, 81:559-575.

13. Lawrence R, Day-Williams AG, Elliott KS, Morris AP, Zeggini E: CCRaVAT and QuTie-enabling analysis of rare variants in large-scale case control and quantitative trait association studies. BMC Bioinform 2010, 11:527.

14. Kanehisa M, Goto S: KEGG: Kyoto Encyclopedia of Genes and Genomes. Nucleic Acids Res 2000, 28:27-30.

15. Ingenuity Systems: Ingenuity Knowledge Base.[http://www.ingenuity.com]

16. Ashburner M, Ball CA, Blake JA, Botstein D, Butler H, Cherry JM, Davis AP, Dolinski K, Dwight SS, Eppig JT, et al: Gene ontology: tool for the unification of biology. Nat Genet 2000, 25:25-29.

17. Salford Systems: TreeNet 1.0 stochastic gradient boosting. San Diego, CA, Salford Systems; 2002.

18. Friedman $\mathrm{JH}$ : Greedy function approximation: a gradient boosting machine. Ann Stat 2001, 29:1189-1232.

19. Hastie T, Tibshirani R, Friedman JH: The elements of statistical learning New York, Springer; 22 2009, 337-384.

20. Hirschhorn JN: Genomewide association studies: illuminating biologic pathways. New Engl J Med 2009, 360:1699-1701.

doi:10.1186/1753-6561-5-S9-S94

Cite this article as: Cherkas et al:: Rare variant collapsing in conjunction with mean log $p$-value and gradient boosting approaches applied to Genetic Analysis Workshop 17 data. BMC Proceedings 2011 5(Suppl 9):S94.

\section{Submit your next manuscript to BioMed Central and take full advantage of:}

- Convenient online submission

- Thorough peer review

- No space constraints or color figure charges

- Immediate publication on acceptance

- Inclusion in PubMed, CAS, Scopus and Google Scholar

- Research which is freely available for redistribution
Ciomed Central 